\title{
Quality of Life and Awareness of Hypertension Among Hypertensive Patients in Saudi Arabia
}

Sulaiman A. Alshammari ${ }^{1}$, Anwar N. Alajmi ${ }^{2}$, Rema A. Albarrak ${ }^{2}$, Alaa B. Alaqil ${ }^{2}$, Ghaida K. Alsaeed ${ }^{2}$, Muneerah Z. Alzayed ${ }^{2}$, Hamdan N. Alajami ${ }^{3}$, Jaffer B. Baqar ${ }^{4}$, Sheraz Ali ${ }^{5}$

1. Family and Community Medicine, King Saud University, Riyadh, SAU 2. College of Medicine, King Saud University, Riyadh, SAU 3. Pharmaceutical Care Services, King Saud Medical City, Riyadh, SAU 4. Statistics, University of Karachi, Karachi, PAK 5. School of Pharmacy and Pharmacology, University of Tasmania, Hobart, AUS

Corresponding author: Sheraz Ali, sheraz.ali@utas.edu.au

\section{Abstract}

\section{Background and aim}

Hypertension possesses significant public health challenges for both developing and developed economies. Therefore, it is crucial to evaluate the awareness of hypertension and health-related quality of life (HRQoL) among patients with hypertension. This study aims to evaluate the knowledge of hypertension and HRQoL among hypertensive patients.

\section{Materials and methods}

A cross-sectional study using an anonymous questionnaire was conducted over a period of 4 months (November 2020 to February 2021) in Riyadh, Kingdom of Saudi Arabia (KSA). This study included 437 questionnaires submitted by the hypertensive population.

\section{Results}

A total of 437 participants were included in this study, with $55.4 \%$ being males $(n=242)$. The majority $(85.1 \%)$ were aware of the normal values of blood pressure. Many participants had no problems with mobility, personal care, usual activities, pain or discomfort, and anxiety or depression. Age groups showed a significant association with mobility and usual activities. The knowledge of hypertension was significantly associated with HRQoL.

\section{Conclusion}

This study found that majority of the hypertensive patients were aware of the standard values of blood pressure. HRQoL concerning physical functioning and general health is found to be good based on the EuroQol visual analogue scale (EQ VAS) in the hypertensive population who are aware of their condition. This study reported a weak yet significant association between hypertension-related knowledge and HRQOL scores. Several factors can affect the HRQoL of the hypertensive population including gender, education, occupation, and income status.

Review began 04/22/2021 Review ended 04/30/2021 Published 05/06/2021

\section{๑) Copyright 2021}

Alshammari et al. This is an open access article distributed under the terms of the Creative Commons Attribution License CC-BY 4.0., which permits unrestricted use, distribution, and reproduction in any medium, provided the original author and source are credited.
Categories: Family/General Practice, Internal Medicine, Epidemiology/Public Health

Keywords: hypertension in the global context, heath related quality of life, patient knowledge, clinic blood pressure, keywords awareness

\section{Introduction}

Hypertension (defined as systolic blood pressure above $140 \mathrm{mmHg}$ with a diastolic blood pressure of less than $90 \mathrm{mmHg}$ ) is quantitatively the most important modifiable risk factor of cardiovascular and kidney disease, and a leading risk factor for mortality [1]. It causes significant public health challenge for both developing and developed economies [2]. According to the World Health Organization, an estimated 1.13 billion people worldwide have hypertension [3]. Globally, seven million people die each year because of hypertension [4]. The prevalence of hypertension in the Kingdom of Saudi Arabia (KSA) is $25.5 \%$ among those aged 15-64 years [5-7]. The most recent study revealed that the prevalence of hypertension among the Saudi population is $31.4 \%$ [8]. A nationwide survey in the KSA revealed that $57.8 \%$ of those with hypertension were not aware of their condition [9]. Lack of information and poor self-care practice increases the burden of hypertension and are a major challenge in controlling hypertension [10]. In the KSA, hypertension is an important public health problem and has become a leading risk factor for death [11].

Health-related quality of life (HRQoL) is defined as "an individual's or a group's perceived physical and mental health over time” [12]. HRQoL is a multi-dimensional concept and critical indicator of an individual's overall health as it captures information on the physical and mental health state of individuals and on the impact of health status on quality of life $[13,14]$. Hypertension adversely affects a patient's 
quality of life [15]. Previous studies have also shown a significant reduction in the HRQoL in hypertensive population to be associated with patient health comorbidities, knowledge of diagnosis, and adverse effects from medications [16].

Given the high prevalence of hypertension and lack of awareness, the impact on patient's quality of life is inevitable. There is a growing demand to increase awareness among patients about chronic diseases [17]. Several strategies have earlier been implemented to improve patient's knowledge including patient groups, published scientific literature, specialists' clinics, and the uptake of information technology [18]. The provision of disease-related knowledge to the population is regarded as a good practice; however, the impact of this knowledge on HRQoL is uncertain. Furthermore, no specific information about the impact of hypertension-related awareness on HRQoL for the hypertensive Saudi population exists. Therefore, this study aims to evaluate the knowledge of hypertension and HRQoL among hypertensive patients. In addition, we also examined the association between HRQoL and hypertension-related knowledge among the hypertensive population in the KSA. The study will help determine the factors compromising the quality of the life and facilitate the designing and implementation of customized educational programs for the hypertensive population, thereby improving the HRQoL.

\section{Materials And Methods}

This cross-sectional study was conducted among hypertensive Saudi patients from November 2020 to February 2021 at the outpatient clinic of the King Saud University Medical City, a tertiary care setting in Riyadh, KSA. This medical city serves a broad range of patients drawn from a large population, several of whom present with complex medical comorbidities.

Patients aged 18 years and over, with a confirmed diagnosis (blood pressure reading was taken on two separate occasions that is greater than $140 / 90 \mathrm{mmHg}$ ) of essential hypertension, who have been using antihypertensive agents for the previous three months were included in the study. Patients aged $<18$ and > 80 years, having co-morbidities, expatriates from other countries, and pregnant women were excluded.

A 21-item self-administered questionnaire for assessing the knowledge of hypertension was distributed among hypertensive patients at the King Saud University Medical City. This questionnaire was adopted from previous studies [19]. The questionnaire was used to collect demographics, duration of disease and assess hypertensive patient's knowledge toward causes, treatment, and management of hypertension. The assessment of the knowledge was primarily based on the knowledge about normal values of blood pressure.

Data for the quality of life was collected using EuroQol five-dimensions - 3-level (EQ-5D-3L), the European quality of life scale [20]. EQ-5D-3L consists of two sections: the EQ-5D descriptive system and the EQ visual analogue scale (EQ VAS). The EQ-5D-3L descriptive system comprises the following five dimensions: mobility, self-care, usual activities, pain or discomfort, and anxiety or depression. Each dimension has the following three levels: no problems, some or moderate problems, and extreme problems. The hypertensive patient was asked to indicate his or her health state by selecting the box next to the most appropriate statement in each of the five dimensions. The EQ VAS records the patient's self-rated health on a $20 \mathrm{~cm}$ vertical visual analogue scale with two distinct endpoints such as "Best imaginable health state" for a score of 100 and "Worst imaginable health state" for a score of 0 . The VAS is used as a quantitative measure of health outcome that reflects the subject's own judgement. All instruments were pre-tested for reliability and validity. The translated Arabic version of EQ-5D-3L was provided by the Euroqol after registering this study on Euroqol's website. All participants were briefed about the purpose of the study and were also provided with a study information sheet; Informed consent was obtained from all the study participants. This study was initiated after the approval of the Institutional Review Board of King Saud University Medical City (Reference number: E-20-5457).

In order to elicit a response in 50\% of respondents regarding the knowledge of hypertension, with a $95 \%$ confidence level and 5\% margin of error, a sample size of 377 was estimated. The sample size was calculated by an online sample size calculator. Data analysis was carried out using the SPSS software, version 24 (IBM. Armonk, NY: IBM Corp). Numerical data were presented with mean and standard deviation whereas categorical data in frequencies and percentages. Independent samples' t-test and analysis of variance (ANOVA) were applied for two and more than two means difference, respectively. EQ-5D-3L scores for five dimensions and mean EQ VAS scores were also compared for age and gender. Pearson correlation was used to explore any correlations between knowledge and HRQoL. Correlations were interpreted using the following criteria: 0 to 0.25 = weak correlation, 0.25 to 0.5 = fair correlation, 0.5 to $0.75=$ good correlation and $>0.75=$ excellent correlation [21]. A P-value of $<0.05$ was taken as the level of significance between responses.

\section{Results}

A total of 437 questionnaires were returned by the hypertensive patients, and the response rate was $87.4 \%$. The majority of the patients belong to the age group of 48 years and over, with $55.4 \%$ being males $(n=242)$. Nearly $47 \%$ of the study participants had a bachelor's degree. Twenty-six percent $(n=115)$ had a monthly income of more than 15,000 Saudi riyals. Ninety-seven percent of the study participants resided in urban 


\section{Cureus}

settings and 3\% were from rural areas. Nearly 36\% had a duration of hypertension for more than 15 years. There was a significant association in EQ-VAS scores with respect to gender, qualification, occupation, monthly income, and the duration of disease. The mean EQ-VAS score $(77.1 \pm 17.6)$ indicated good HRQoL in our study participants (Table 1 ).

\begin{tabular}{|c|c|c|c|}
\hline Variables & n (n\%) & EQ VAS score (mean \pm SD) & P-value \\
\hline \multicolumn{4}{|l|}{ Age groups (years) } \\
\hline $18-27$ & $03(0.7)$ & $80 \pm 10$ & \multirow{4}{*}{0.203} \\
\hline 28-37 & $12(2.7)$ & $81.7 \pm 11.9$ & \\
\hline $38-47$ & $57(13)$ & $81.1 \pm 18.1$ & \\
\hline $48 \&$ above & $365(83.5)$ & $76.3 \pm 17.7$ & \\
\hline \multicolumn{4}{|l|}{ Gender } \\
\hline Male & 242 (55.4) & $80.5 \pm 15.3$ & \multirow{2}{*}{$<0.001$} \\
\hline Female & $195(44.6)$ & $72.7 \pm 19.3$ & \\
\hline \multicolumn{4}{|l|}{ Education } \\
\hline No formal education & $37(8.5)$ & $62.2 \pm 25.4$ & \multirow{5}{*}{$<0.001$} \\
\hline Primary & 58 (13.3) & $76.2 \pm 17.4$ & \\
\hline Intermediate & $84(19.2)$ & $74.8 \pm 17.2$ & \\
\hline Bachelors & 203 (46.5) & $79.6 \pm 15.6$ & \\
\hline Masters & $55(12.6)$ & $82 \pm 13.5$ & \\
\hline \multicolumn{4}{|l|}{ Occupation } \\
\hline Retired & $146(33.4)$ & $77.7 \pm 16.1$ & \multirow{4}{*}{$<0.001$} \\
\hline Jobless & $146(33.4)$ & $72.5 \pm 20.1$ & \\
\hline Government job & $136(31.1)$ & $81.3 \pm 15.4$ & \\
\hline Businessman & $09(2.1)$ & $77.8 \pm 15.6$ & \\
\hline \multicolumn{4}{|l|}{ Income } \\
\hline Nill & $91(20.8)$ & $72.8 \pm 19.6$ & \multirow{5}{*}{$<0.001$} \\
\hline Less than 5,000 & 59 (13.5) & $68.9 \pm 20.2$ & \\
\hline $5,000-10,000$ & $88(20.1)$ & $79.7 \pm 16.8$ & \\
\hline $10,001-15,000$ & 84 (19.2) & $80.7 \pm 13.8$ & \\
\hline Above 15,000 & $115(26.3)$ & $80 \pm 15.6$ & \\
\hline \multicolumn{4}{|l|}{ Locality } \\
\hline Urban & $424(97)$ & $77.3 \pm 17.4$ & \multirow{2}{*}{0.104} \\
\hline Rural & $13(3)$ & $69.2 \pm 22.2$ & \\
\hline \multicolumn{4}{|c|}{ Duration of disease (years) } \\
\hline$<1$ & $15(3.4)$ & $75.3 \pm 22.0$ & \multirow{5}{*}{0.024} \\
\hline $1-3$ & $42(9.6)$ & $81.4 \pm 14.6$ & \\
\hline 4-5 & 61 (14) & $82.3 \pm 16.4$ & \\
\hline $6-15$ & $162(37.1)$ & $76.4 \pm 16.8$ & \\
\hline$>15$ & 157 (35.9) & $74.7 \pm 18.7$ & \\
\hline
\end{tabular}




\section{Cureus}

\section{TABLE 1: Mean EQ VAS scores among study demographics.}

EQ VAS: EuroQol visual analogue scale.

More than half of the participants (55.4\%) were not aware of the basic definition of hypertension; however, the majority (85.1\%) were aware of the normal values of blood pressure (Table 2). More than $80 \%$ correctly identified smoking, fatty food, and obesity as risk factors of hypertension. Many study participants (93.4\%) were aware that hypertension can lead to other life-threatening diseases.

\begin{tabular}{|c|c|c|c|c|c|c|c|}
\hline \multirow{3}{*}{ Variables } & \multirow{3}{*}{$\begin{array}{l}\text { Overall } \\
(n=437)\end{array}$} & \multicolumn{3}{|c|}{ Age groups (years) } & \multicolumn{3}{|l|}{ Gender } \\
\hline & & $18-47$ & $48 \&$ above & & Male & Female & \\
\hline & & $(n=72)$ & $(n=365)$ & & $(n=242)$ & $(n=195)$ & \\
\hline \multicolumn{8}{|c|}{ Q1: Know the normal values of blood pressure } \\
\hline Yes & $372(85.1)$ & $62(86.1)$ & $310(84.9)$ & \multirow{2}{*}{0.797} & $222(91.7)$ & $150(76.9)$ & \multirow{2}{*}{$<0.001$} \\
\hline No & 65 (14.9) & $10(13.9)$ & $55(15.1)$ & & $20(8.3)$ & $45(23.1)$ & \\
\hline \multicolumn{8}{|c|}{ Q2: Elevated blood pressure is called hypertension } \\
\hline Yes & $167(38.2)$ & $30(41.7)$ & $137(37.5)$ & \multirow{3}{*}{$<0.001$} & $109(45)$ & $58(29.7)$ & \multirow{3}{*}{0.001} \\
\hline No & $28(6.4)$ & $12(16.7)$ & $16(4.4)$ & & $10(4.1)$ & $18(9.2)$ & \\
\hline Don't know & $242(55.4)$ & $30(41.7)$ & $212(58.1)$ & & $123(50.8)$ & $119(61)$ & \\
\hline \multicolumn{8}{|c|}{ Q3: Hypertension can progress with age } \\
\hline Yes & $213(48.7)$ & $33(45.8)$ & $180(49.3)$ & \multirow{3}{*}{0.857} & $126(52.1)$ & $87(44.6)$ & \multirow{3}{*}{0.251} \\
\hline No & $36(8.2)$ & $6(8.3)$ & $30(8.2)$ & & $17(7)$ & $19(9.7)$ & \\
\hline Don't know & $188(43)$ & $33(45.8)$ & $155(42.5)$ & & 99 (40.9) & $89(45.6)$ & \\
\hline \multicolumn{8}{|c|}{ Q4: Both genders have equal chance of developing hypertension } \\
\hline Yes & $107(24.5)$ & $22(30.6)$ & $85(23.3)$ & \multirow{3}{*}{0.423} & $52(21.5)$ & $55(28.2)$ & \multirow{3}{*}{0.072} \\
\hline No & $113(25.9)$ & $17(23.6)$ & $96(26.3)$ & & $72(29.8)$ & $41(21)$ & \\
\hline Don't know & $217(49.7)$ & $33(45.8)$ & $184(50.4)$ & & $118(48.8)$ & $99(50.8)$ & \\
\hline \multicolumn{8}{|c|}{ Q5: HTN is a treatable condition } \\
\hline Yes & $258(59)$ & $38(52.8)$ & $220(60.3)$ & \multirow{3}{*}{0.268} & $144(59.5)$ & $114(58.5)$ & \multirow{3}{*}{0.46} \\
\hline No & $60(13.7)$ & $14(19.4)$ & 46 (12.6) & & $29(12)$ & 31 (15.9) & \\
\hline Don’t know & $119(27.2)$ & $20(27.8)$ & $99(27.1)$ & & $69(28.5)$ & $50(25.6)$ & \\
\hline \multicolumn{8}{|c|}{ Q6: The older a person is, greater risk of having hypertension } \\
\hline Yes & 345 (78.9) & $56(77.8)$ & $289(79.2)$ & \multirow{3}{*}{0.097} & $188(77.7)$ & $157(80.5)$ & \multirow{3}{*}{0.768} \\
\hline No & $31(7.1)$ & 9 (12.5) & $22(6)$ & & $18(7.4)$ & $13(6.7)$ & \\
\hline Don't know & $61(14)$ & $7(9.7)$ & $54(14.8)$ & & 36 (14.9) & $25(12.8)$ & \\
\hline \multicolumn{8}{|c|}{ Q7: Smoking is a risk factor for hypertension } \\
\hline Yes & $357(81.7)$ & $68(94.4)$ & $289(79.2)$ & \multirow{3}{*}{0.008} & $199(82.2)$ & $158(81)$ & \multirow{3}{*}{0.867} \\
\hline No & $10(2.3)$ & 0 & $10(2.7)$ & & $6(2.5)$ & $4(2.1)$ & \\
\hline Don't know & 70 (16) & $4(5.6)$ & $66(18.1)$ & & 37 (15.3) & 33 (16.9) & \\
\hline \multicolumn{8}{|c|}{ Q8: Eating fatty food is a risk factor for hypertension } \\
\hline Yes & 406 (92.9) & $72(100)$ & $334(91.5)$ & & $229(94.6)$ & $177(90.8)$ & \\
\hline
\end{tabular}




\section{Cureus}

No

$$
05(1.1) \quad 0
$$

5 (1.4)

0.037

3 (1.2)

2 (1)

0.199

Don't know

$26(5.9)$

$26(7.1)$

$10(4.1)$

$16(8.2)$

Q9: Overweight increases risk for hypertension

$\begin{array}{llll}\text { Yes } & 406(92.9) & 72(100) & 334(91.5) \\ \text { No } & 06(1.4) & 0 & 6(1.6) \\ \text { Don't know } & 25(5.7) & 0 & 25(6.9)\end{array}$

0.037

226 (93.4)

$180(92.3)$

$5(2.1)$

$1(0.5)$

0.200

Q10: Regular physical activity lowers a chance of hypertension

$\begin{array}{llll}\text { Yes } & 384(87.9) & 68(94.4) & 316(86.6) \\ \text { No } & 16(3.7) & 2(2.8) & 14(3.8) \\ \text { Don’t know } & 37(8.5) & 2(2.8) & 35(9.6)\end{array}$

0.142

$11(4.5)$

14 (7.2)

Q11: Eating more salt has no effect on blood pressure

$\begin{array}{llll}\text { Yes } & 30(6.9) & 4(5.6) & 26(7.1) \\ \text { No } & 374(85.6) & 63(87.5) & 311(85.2) \\ \text { Don't know } & 33(7.6) & 5(6.9) & 28(7.7)\end{array}$

Q12: Dietary approaches to reduce hypertension do no good

\begin{tabular}{|c|c|c|c|c|c|c|c|}
\hline Yes & $82(18.8)$ & $16(22.2)$ & $66(18.1)$ & \multirow{3}{*}{0.129} & 37 (15.3) & $45(23.1)$ & \multirow{3}{*}{0.116} \\
\hline No & $300(68.6)$ & $52(72.2)$ & 248 (67.9) & & $173(71.5)$ & $127(65.1)$ & \\
\hline Don’t know & $55(12.6)$ & $4(5.6)$ & $51(14)$ & & $32(13.2)$ & $23(11.8)$ & \\
\hline \multicolumn{8}{|c|}{ Q13: White meat is as good as red meat in hypertension } \\
\hline Yes & $130(29.7)$ & $24(33.3)$ & $106(29)$ & \multirow{3}{*}{0.686} & $73(30.2)$ & $57(29.2)$ & \multirow{3}{*}{0.005} \\
\hline No & $176(40.3)$ & $26(36.1)$ & $150(41.1)$ & & $111(45.9)$ & $65(33.3)$ & \\
\hline Don’t know & $131(30)$ & $22(30.6)$ & $109(29.9)$ & & $58(24)$ & $73(37.4)$ & \\
\hline \multicolumn{8}{|c|}{ Q14: Medication alone can control hypertension } \\
\hline Yes & $53(12.1)$ & $5(6.9)$ & 48 (13.2) & \multirow{3}{*}{0.123} & 32 (13.2) & 21 (10.8) & \multirow{3}{*}{0.624} \\
\hline No & $350(80.1)$ & $64(88.9)$ & $286(78.4)$ & & $193(79.8)$ & $157(80.5)$ & \\
\hline Don't know & $34(7.8)$ & $3(4.2)$ & $31(8.5)$ & & $17(7)$ & $17(8.7)$ & \\
\hline \multicolumn{8}{|c|}{ Q15: Hypertension can lead to other life-threatening diseases } \\
\hline Yes & $408(93.4)$ & $70(97.2)$ & 338 (92.6) & \multirow{3}{*}{0.342} & $230(95)$ & $178(91.3)$ & \multirow{3}{*}{0.139} \\
\hline No & $02(0.5)$ & 0 & $2(0.5)$ & & 0 & $2(1)$ & \\
\hline Don't know & $27(6.2)$ & $2(2.8)$ & $25(6.9)$ & & $12(5)$ & $15(7.7)$ & \\
\hline
\end{tabular}

TABLE 2: Comparison of responses about hypertension knowledge among age groups and gender.

Table 3 and Table 4 report HRQoL scores among study participants. Regarding the five dimensions of EQ5D-3L, many participants had no problems with mobility (63.6\%), personal care (93.4\%), usual activities (74.4\%), pain or discomfort (54.5\%), and anxiety or depression (65.7\%); however, age groups showed a significant association with mobility and usual activities. Both mobility and usual activities had a significant association with age and gender. In contrast, personal care $(\mathrm{P}<0.001)$, pain or discomfort $(\mathrm{P}<0.001)$, and anxiety or depression $(\mathrm{P}=0.009)$ showed a significant association with gender (Table 3 ). 


\section{Cureus}

\begin{tabular}{|c|c|c|c|c|c|c|c|}
\hline \multirow{3}{*}{ Variables } & \multirow{3}{*}{$\begin{array}{l}\text { Overall } \\
(n=437)\end{array}$} & \multicolumn{3}{|c|}{ Age groups (years) } & \multicolumn{3}{|l|}{ Gender } \\
\hline & & $18-47$ & $\begin{array}{l}40 \propto \alpha \\
\text { above }\end{array}$ & P- & Male & Female & P-value \\
\hline & & $(n=72)$ & $(n=365)$ & & $(n=242)$ & $(n=195)$ & \\
\hline \multicolumn{8}{|l|}{ Mobility } \\
\hline I have no problems with walking around & $\begin{array}{l}278 \\
(63.6)\end{array}$ & $\begin{array}{l}62 \\
(86.1)\end{array}$ & $216(59.2)$ & \multirow{3}{*}{$<0.001$} & $184(76)$ & $94(48.2)$ & \multirow{3}{*}{$<0.001$} \\
\hline I have some problems with walking around & $\begin{array}{l}154 \\
(35.2)\end{array}$ & $8(11.1)$ & $146(40)$ & & $54(22.3)$ & $\begin{array}{l}100 \\
(51.3)\end{array}$ & \\
\hline I am confined to bed & $5(1.1)$ & $2(2.8)$ & $3(0.8)$ & & $4(1.7)$ & $1(0.5)$ & \\
\hline \multicolumn{8}{|l|}{ Personal care } \\
\hline I have no problems with washing or dressing myself & $\begin{array}{l}408 \\
(93.4)\end{array}$ & $\begin{array}{l}66 \\
(91.7)\end{array}$ & $342(93.7)$ & \multirow{3}{*}{0.384} & $\begin{array}{l}233 \\
(96.3)\end{array}$ & $\begin{array}{l}175 \\
(89.7)\end{array}$ & \multirow{3}{*}{0.015} \\
\hline I have some problems with washing or dressing myself & $20(4.6)$ & $3(4.2)$ & $17(4.7)$ & & $5(2.1)$ & $15(7.7)$ & \\
\hline I am unable to wash or dress myself & $9(2.1)$ & $3(4.2)$ & $6(1.6)$ & & $4(1.7)$ & $5(2.6)$ & \\
\hline \multicolumn{8}{|c|}{ Usual activities (e.g., work, study, housework, family or leisure activities) } \\
\hline I have no problems with performing my usual activities & $\begin{array}{l}325 \\
(74.4)\end{array}$ & $\begin{array}{l}65 \\
(90.3)\end{array}$ & $260(71.2)$ & & $213(88)$ & $\begin{array}{l}112 \\
(57.4)\end{array}$ & \multirow{3}{*}{$<0.001$} \\
\hline $\begin{array}{l}\text { I have some problems with performing my usual } \\
\text { activities }\end{array}$ & $95(21.7)$ & $6(8.3)$ & $89(24.4)$ & 0.003 & $22(9.1)$ & 73 (37.4) & \\
\hline I am unable to perform my usual activities & $17(3.9)$ & $1(1.4)$ & $16(4.4)$ & & $7(2.9)$ & $10(5.1)$ & \\
\hline \multicolumn{8}{|l|}{ Pain/discomfort } \\
\hline I have no pain or discomfort & $\begin{array}{l}238 \\
(54.5)\end{array}$ & $\begin{array}{l}46 \\
(63.9)\end{array}$ & $192(52.6)$ & \multirow{3}{*}{0.184} & $\begin{array}{l}172 \\
(71.1)\end{array}$ & $66(33.8)$ & \multirow{3}{*}{$<0.001$} \\
\hline I have moderate pain or discomfort & $\begin{array}{l}186 \\
(42.6)\end{array}$ & $\begin{array}{l}25 \\
(34.7)\end{array}$ & $161(44.1)$ & & $67(27.7)$ & $119(61)$ & \\
\hline I have extreme pain or discomfort & $13(3)$ & $1(1.4)$ & $12(3.3)$ & & $3(1.2)$ & $10(5.1)$ & \\
\hline \multicolumn{8}{|l|}{ Anxiety/depression } \\
\hline I am not anxious or depressed & $\begin{array}{l}287 \\
(65.7)\end{array}$ & $\begin{array}{l}46 \\
(63.9)\end{array}$ & 241 (66) & & $\begin{array}{l}174 \\
(71.9)\end{array}$ & $\begin{array}{l}113 \\
(57.9)\end{array}$ & \multirow{3}{*}{0.009} \\
\hline I am moderately anxious or depressed & $\begin{array}{l}142 \\
(32.5)\end{array}$ & $\begin{array}{l}24 \\
(33.3)\end{array}$ & $118(32.3)$ & 0.785 & $64(26.4)$ & $78(40)$ & \\
\hline I am extremely anxious or depressed & $8(1.8)$ & $2(2.8)$ & $6(1.6)$ & & $4(1.7)$ & $4(2.1)$ & \\
\hline
\end{tabular}

TABLE 3: Association of five dimensions of EQ-5D-3L with age groups and gender.

EQ-5D-3L: EuroQol five-dimensions-three-level.

\begin{tabular}{|c|c|c|c|c|c|c|c|c|c|c|c|c|}
\hline \multirow{2}{*}{ Variables } & \multicolumn{2}{|c|}{ EQ VAS score } & \multicolumn{2}{|c|}{ Mobility } & \multicolumn{2}{|c|}{ Personal care } & \multicolumn{2}{|c|}{ Usual activities } & \multicolumn{2}{|c|}{ Pain/discomfort } & \multicolumn{2}{|c|}{ Anxiety/depression } \\
\hline & Mean \pm SD & P-value & $\mathrm{r}$ & P-value & $\mathrm{r}$ & P-value & $r$ & P-value & $r$ & P-value & $\mathrm{r}$ & P-value \\
\hline \multicolumn{13}{|c|}{ Know the normal values of blood pressure } \\
\hline Yes & $78.4 \pm 16.6$ & $<0.001$ & 0.17 & $<0.001$ & 0.2 & $<0.001$ & 0.2 & $<0.001$ & 0.2 & $<0.001$ & 0.14 & 0.004 \\
\hline No & $69.3 \pm 21.0$ & & & & & & & & & & & \\
\hline
\end{tabular}




\section{Cureus}

Elevated blood pressure is called hypertension

Yes

$0.02 \quad 0.67$

$0.02 \quad 0.615$

$0.04 \quad 0.4$

$0.08 \quad 0.085$

0.04

0.466

No

$84.6 \pm 12.3$

Hypertension can progress with age

$\begin{array}{llllllllllllll}\text { Yes } & 77.3 \pm 16.8 & 0.736 & 0.03 & 0.486 & 0.01 & 0.885 & 0.03 & 0.491 & 0.04 & 0.434 & -0.07 & 0.137\end{array}$

No $\quad 78.4 \pm 15.4$

Both genders have equal chance of developing hypertension

$\begin{array}{lllllllllllll}\text { Yes } & 78.2 \pm 17.4 & 0.564 & 0.06 & 0.196 & 0.07 & 0.146 & 0.05 & 0.325 & 0.07 & 0.122 & -0.01 & 0.88 \\ \text { No } & 76.9 \pm 16.5 & & & & & & & & & & & \end{array}$

Hypertension is a treatable condition

$\begin{array}{lllllllllllll}\text { Yes } & 77.5 \pm 17.9 & 0.552 & -0.03 & 0.545 & -0.02 & 0.632 & 0.03 & 0.491 & 0.03 & 0.483 & -0.03 & 0.578\end{array}$

No $\quad 76.0 \pm 17.8$

The older a person is, the greater risk of having hypertension

$\begin{array}{llllllllllllll}\text { Yes } & 77.2 \pm 17.6 & 0.509 & 0.04 & 0.411 & -0.01 & 0.938 & 0.05 & 0.342 & 0.02 & 0.678 & -0.04 & 0.36\end{array}$

No $\quad 79.4 \pm 11.8$

Smoking is a risk factor for hypertension

$\begin{array}{llllllllllllll}\text { Yes } & 78.3 \pm 16.9 & 0.058 & 0.21 & <0.001 & -0.01 & 0.861 & 0.11 & 0.024 & 0.14 & 0.003 & -0.01 & 0.793\end{array}$

No $\quad 68.0 \pm 20.4$

Eating fatty food is a risk factor for hypertension

$\begin{array}{llllllllllllll}\text { Yes } & 77.2 \pm 17.3 & 0.54 & 0.09 & 0.073 & 0.04 & 0.464 & 0.04 & 0.361 & 0.05 & 0.297 & -0.09 & 0.064\end{array}$

No $\quad 82.0 \pm 14.8$

Overweight increases risk for hypertension

$\begin{array}{lllllllllllll}\text { Yes } & 77.6 \pm 17.2 & 0.123 & 0.07 & 0.164 & -0.07 & 0.124 & 0.08 & 0.095 & 0.06 & 0.22 & 0.04 & 0.36\end{array}$

No $\quad 66.7 \pm 15.1$

Regular physical activity lowers a chance of hypertension

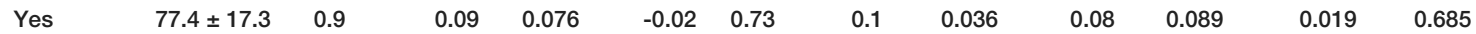

No $\quad 76.9 \pm 17.4$

Eating more salt has no effect on blood pressure

$\begin{array}{lllllllllllll}\text { Yes } & 79.7 \pm 17.5 & 0.418 & 0.06 & 0.207 & 0.02 & 0.698 & 0.09 & 0.075 & 0.13 & 0.008 & 0.02 & 0.739\end{array}$

No $\quad 77.1 \pm 17.1$

Dietary approaches to reduce hypertension do no good

$\begin{array}{llllllllllllll}\text { Yes } & 74.9 \pm 17.4 & 0.17 & 0.01 & 0.816 & -0.07 & 0.131 & -0.02 & 0.757 & -0.04 & 0.4 & -0.08 & 0.097\end{array}$

No $\quad 77.8 \pm 17.1$

White meat is as good as red meat in hypertension

$\begin{array}{llllllllllllll}\text { Yes } & 77.5 \pm 18.4 & 0.631 & 0.05 & 0.26 & 0.03 & 0.497 & 0.03 & 0.539 & 0.09 & 0.057 & -0.03 & 0.607\end{array}$

No $\quad 78.4 \pm 15.9$

Medication alone can control hypertension

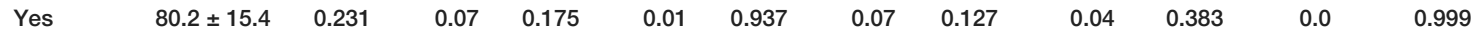

No $\quad 77.2 \pm 17.1$

Hypertension can lead to other life-threatening diseases 


\section{Cureus}

\begin{tabular}{|c|c|c|c|c|c|c|c|c|c|c|c|c|}
\hline Yes & $77.3 \pm 17.1$ & 0.523 & -0.01 & 0.919 & -0.03 & 0.501 & 0.11 & 0.023 & 0.07 & 0.152 & -0.06 & 0.22 \\
\hline & $.0 \pm 7.1$ & & & & & & & & & & & \\
\hline
\end{tabular}

TABLE 4: Mean EQ VAS score comparison and correlation of EQ-5D levels with responses about hypertension knowledge.

EQ VAS: EuroQol visual analogue scale; EQ-5D: EuroQol five-dimensions.

The score of HRQoL based on EQ-VAS score range from 0 (worst imaginable health state) to 100 (best imaginable health state) (Figure 1).

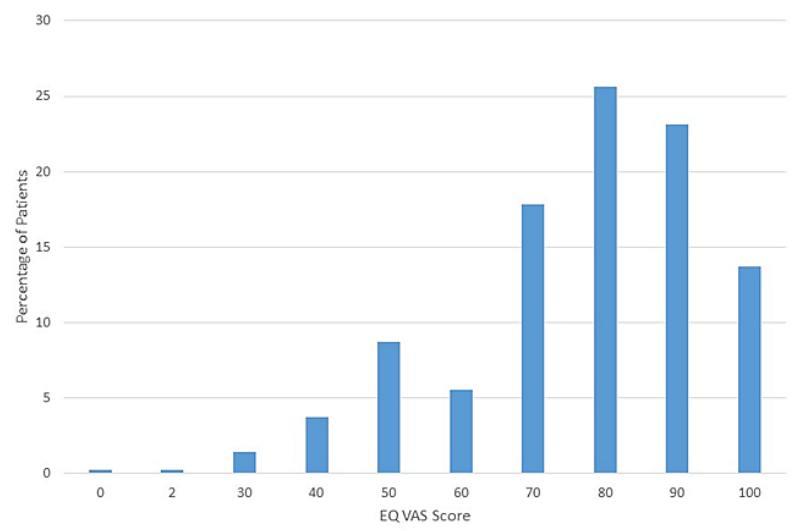

FIGURE 1: EQ VAS health index of study participants.

EQ VAS: EuroQol visual analogue scale.

Table 4 showed the correlation between HRQoL and knowledge of hypertension. The study findings revealed that the main variables of the knowledge about hypertension such as normal values $(\mathrm{P}<0.001)$ and the definition ( $\mathrm{P}=0.028$ ) of hypertension were significantly associated with HRQoL (mean VAS score) (Table 4). Pearson correlation test showed that the knowledge about normal values of blood pressure and HRQoL scores had a weak correlation. The knowledge about the definition of hypertension and HRQoL scores also showed a weak correlation.

\section{Discussion}

To our knowledge, this is the first study conducted in the KSA using EQ-5D-3L to measure HRQoL among hypertensive patients visiting outpatient clinics. Moreover, the relationship between the knowledge of hypertension and HRQoL has not been explored among the Saudi population. This study reports that many hypertensive patients, particularly those living in urban areas, had knowledge about the standard values of blood pressure. Similarly, the findings of this study highlight that the knowledge of hypertension (based on the normal values of blood pressure) is significantly associated with HRQoL, although a weak correlation. This finding is consistent with that of other studies conducted in hypertensive population in Pakistan [22]; however, our study is relatively larger in sample size, and also informs about individualized factors affecting HRQoL in hypertensive patients.

Previous studies provide inconsistent findings ranging from weak to strong correlation between the quality of life among hypertensive population and adherence to treatment $[23,24]$. The differences in the findings are probably due to the measurement of HRQoL using different instruments such as the World Health Organization Quality of Life Instrument (WHOQOL-BREF), EQ-5D-3L, Short Form 36 (SF36), or SF-12 [25]. Our results in the outpatient study population indicate that the HRQoL is good in the majority of hypertensive patients who are aware of the normal values of blood pressure. Education has been recognized as an important determinant of HRQoL; patients with higher educational levels usually report better HRQoL [26]. Nearly $60 \%$ of the hypertensive participants in our study had higher education. Previous studies reported better HRQoL among highly educated hypertensive patients [27]. Patients with higher education 
demonstrate good physical function through acquiring knowledge about appropriate health practices [28]. The results of this study also refute the assumption that disease-related awareness can decrease HRQoL particularly in patients suffering from chronic conditions such as hypertension and diabetes mellitus, due to their impact on psychosocial domains [29]. Likewise, the provision of education programs in previous studies demonstrated either a decline or no improvement in HRQoL scores [30]. Numerous methods have been utilized to improve patient knowledge such as specialist clinics, scientific literature, and information technology [18].

Strengths of our study include patients with a confirmed diagnosis of essential hypertension who have been using antihypertensive agents for the previous three months, the EQ-5D-3L questionnaire which is the European quality of life scale, and exclusion of complications of hypertension that might reduce HRQoL. In addition, we included a large sample of hypertensive patients, and determined individualized factors affecting HRQoL in the hypertensive population. There were some limitations to this study. First, this study was cross-sectional and limited in its capacity to inform about causality. It was conducted at a single tertiary care setting in the KSA, the results cannot be generalized to the entire population. Therefore, these results need to be validated by a multicentre longitudinal study across tertiary care settings in the Kingdom.

\section{Conclusions}

This study found that majority of the hypertensive patients were aware of the standard values of blood pressure. HRQoL concerning physical functioning and general health found to be good based on EQ VAS in the hypertensive population who are aware of their condition. This study reported a weak yet significant association between hypertension-related knowledge and HRQoL scores. Several factors can affect the HRQoL of the hypertensive population including gender, education, occupation, and income status. The quality of life among hypertensive patients can be improved by conducting educational programs about hypertension at the community level. Future studies can explore the effect of an interdisciplinary education programs on hypertensive patient's outcomes.

\section{Additional Information \\ Disclosures}

Human subjects: Consent was obtained or waived by all participants in this study. King Saud University Medical City issued approval E-20-5457. Animal subjects: All authors have confirmed that this study did not involve animal subjects or tissue. Conflicts of interest: In compliance with the ICMJE uniform disclosure form, all authors declare the following: Payment/services info: All authors have declared that no financial support was received from any organization for the submitted work. Financial relationships: All authors have declared that they have no financial relationships at present or within the previous three years with any organizations that might have an interest in the submitted work. Other relationships: All authors have declared that there are no other relationships or activities that could appear to have influenced the submitted work.

\section{References}

1. Kearney PM, Whelton M, Reynolds K, Muntner P, Whelton PK, He J: Global burden of hypertension: analysis of worldwide data. Lancet. 2005, 365:217-23. 10.1016/S0140-6736(05)17741-1

2. Aldiab A, Shubair MM, Al-Zahrani JM, et al.: Prevalence of hypertension and prehypertension and its associated cardioembolic risk factors; a population based cross-sectional study in Alkharj, Saudi Arabia. BMC Public Health. 2018, 18:1327. 10.1186/s12889-018-6216-9

3. Hypertension. World Health Organization. (2020). https://www.who.int/health-topics/hypertension/.

4. Son PT, Quang NN, Viet NL, et al.: Prevalence, awareness, treatment and control of hypertension in Vietnam-results from a national survey. J Hum Hypertens. 2012, 26:268-80. 10.1038/jhh.2011.18

5. Al-Hamdan N, Saeed A, Kutbi A, Choudhry AJ, Nooh R: Characteristics, risk factors, and treatment practices of known adult hypertensive patients in saudi arabia. Int J Hypertens. 2011, 2010:168739. 10.4061/2010/168739

6. Al-Nozha MM, Abdullah M, Arafah MR, et al.: Hypertension in Saudi Arabia. Saudi Med J. 2007, 28:77-84.

7. Saeed AA, Al-Hamdan NA, Bahnassy AA, Abdalla AM, Abbas MA, Abuzaid LZ: Prevalence, awareness, treatment, and control of hypertension among Saudi adult population: a national survey. Int J Hypertens. 2011, 2011:174135. 10.4061/2011/174135

8. Ahmed AM, Hersi A, Mashhoud W, Arafah MR, Abreu PC, Al Rowaily MA, Al-Mallah MH: Cardiovascular risk factors burden in Saudi Arabia: The Africa Middle East Cardiovascular Epidemiological (ACE) study. J Saudi Heart Assoc. 2017, 29:235-43. 10.1016/j.jsha.2017.03.004

9. El Bcheraoui C, Memish ZA, Tuffaha M, et al.: Hypertension and its associated risk factors in the kingdom of saudi arabia, 2013: a national survey. Int J Hypertens. 2014, 2014:564679. 10.1155/2014/564679

10. Worku Kassahun C, Asasahegn A, Hagos D, Ashenafi E, Tamene F, Addis G, Endalkachew K: Knowledge on hypertension and self-care practice among adult hypertensive patients at University of Gondar Comprehensive Specialized Hospital, Ethiopia, 2019. Int J Hypertens. 2020, 2020:5649165. 10.1155/2020/5649165

11. Memish ZA, Jaber S, Mokdad AH, AlMazroa MA, Murray CJ, Al Rabeeah AA: Burden of disease, injuries, and risk factors in the Kingdom of Saudi Arabia, 1990-2010. Prev Chronic Dis. 2014, 11:E169. 10.5888/pcd11.140176

12. Health-Related Quality of Life (HRQOL. (Cenrers for Disease Control and Prevention). 
https://www.cdc.gov/hrqol/index.htm..

13. Palermo TM, Long AC, Lewandowski AS, Drotar D, Quittner AL, Walker LS: Evidence-based assessment of health-related quality of life and functional impairment in pediatric psychology. J Pediatr Psychol. 2008, 33:983-96. 10.1093/jpepsy/jsn038

14. Revicki DA, Kleinman L, Cella D: A history of health-related quality of life outcomes in psychiatry . Dialogues Clin Neurosci. 2014, 16:127-35. 10.31887/DCNS.2014.16.2/drevicki

15. Wong C, Gerson A, Hooper SR, et al.: Effect of elevated blood pressure on quality of life in children with chronic kidney disease. Pediatr Nephrol. 2016, 31:1129-36. 10.1007/s00467-015-3262-8

16. Soni RK, Porter AC, Lash JP, Unruh ML: Health-related quality of life in hypertension, chronic kidney disease, and coexistent chronic health conditions. Adv Chronic Kidney Dis. 2010, 17:e17-26. 10.1053/j.ackd.2010.04.002

17. Assal JP, Golay A: Patient education in Switzerland: from diabetes to chronic diseases . Patient Educ Couns. 2001, 44:65-9. 10.1016/s0738-3991(01)00105-7

18. Verma S, Tsai HH, Giaffer MH: Does better disease-related education improve quality of life? A survey of IBD patients. Dig Dis Sci. 2001, 46:865-9. 10.1023/a:1010725106411

19. Roca B, Nadal E, Rovira RE, Valls S, Lapuebla C, Lloría N: Usefulness of a hypertension education program . South Med J. 2003, 96:1133-7. 10.1097/01.SMJ.0000053454.67173.48

20. The EuroQol Group: EuroQol--a new facility for the measurement of health-related quality of life . Health Policy. 1990, 16:199-208. 10.1016/0168-8510(90)90421-9

21. Cohen J: Statistical power analysis for the behavioral sciences . Routledge, New York; 1988. 10.4324/9780203771587

22. Saleem F, Hassali MA, Shafie AA, Atif M, ul Haq N, Aljadhey H: Disease related knowledge and quality of life: a descriptive study focusing on hypertensive population in Pakistan. South Med Rev. 2012, 5:47-52.

23. Côté I, Farris K, Feeny D: Is adherence to drug treatment correlated with health-related quality of life? . Qual Life Res. 2003, 12:621-33. 10.1023/a:1025180524614

24. Pippalla RS, Chinburapa V, Duval R, Akula RS: Interrelationships of quality of life, compliance, clinical outcomes and life satisfaction: a cross-sectional study on hypertensive geriatrics. J Clin Pharm Ther. 1997, 22:357-69. 10.1111/j.1365-2710.1997.tb00019.x

25. Ha NT, Duy HT, Le NH, Khanal V, Moorin R: Quality of life among people living with hypertension in a rural Vietnam community. BMC Public Health. 2014, 14:833. 10.1186/1471-2458-14-833

26. Gholami A, Jahromi LM, Zarei E, Dehghan A: Application of WHOQOL-BREF in measuring quality of life in health-care staff. Int J Prev Med. 2013, 4:809-17.

27. Xu X, Rao Y, Shi Z, Liu L, Chen C, Zhao Y: Hypertension impact on health-related quality of life: a crosssectional survey among middle-aged adults in Chongqing, China. Int J Hypertens. 2016, 2016:7404957. 10.1155/2016/7404957

28. Brennan SL, Turrell G: Neighborhood disadvantage, individual-level socioeconomic position, and selfreported chronic arthritis: a cross-sectional multilevel study. Arthritis Care Res. 2012, 64:721-8. 10.1002/acr.21590

29. Korhonen PE, Kivelä SL, Kautiainen H, Järvenpää S, Kantola I: Health-related quality of life and awareness of hypertension. J Hypertens. 2011, 29:2070-4. 10.1097/HJH.0b013e32834bbca7

30. Borgaonkar MR, Townson G, Donnelly M, Irvine EJ: Providing disease-related information worsens healthrelated quality of life in inflammatory bowel disease. Inflamm Bowel Dis. 2002, 8:264-9. 10.1097/00054725200207000-00005 